Review Article

\title{
Genetic Association of CHAT rs3810950 and rs2177369 Polymorphisms with the Risk of Alzheimer's Disease: A Meta-Analysis
}

\author{
Yong Liu, ${ }^{1,2,3}$ Qicong Chen, ${ }^{4}$ Xu Liu, ${ }^{1,5}$ Mengmeng Dou, ${ }^{1,6}$ Silu Li, ${ }^{7}$ Jiahui Zhou, ${ }^{1,6}$ \\ Hong Liu, ${ }^{2}$ Yongfu Wu, ${ }^{1,8}$ and Zunnan Huang ${ }^{1,2,6}$ \\ ${ }^{1}$ Dongguan Scientific Research Center, Guangdong Medical University, Dongguan, Guangdong 523808, China \\ ${ }^{2}$ School of Pharmacy, Guangdong Medical University, Dongguan, Guangdong 523808, China \\ ${ }^{3}$ Key Laboratory for Research and Development of Natural Drugs of Guangdong Province, Zhanjiang, Guangdong 524023, China \\ ${ }^{4}$ Department of Biochemistry and Molecular Biology, Guangxi Medical University, Nanning, Guangxi 530021, China \\ ${ }^{5}$ The Second School of Clinical Medicine, Guangdong Medical University, Dongguan 523808, China \\ ${ }^{6}$ Key Laboratory for Medical Molecular Diagnostics of Guangdong Province, Dongguan, Guangdong 523808, China \\ ${ }^{7}$ School of Basic Medicine, Guangdong Medical University, Dongguan, Guangdong 523808, China \\ ${ }^{8}$ Affiliated Hospital of Guangdong Medical University, Zhanjiang, Guangdong 524023, China
}

Correspondence should be addressed to Yongfu Wu; wyf163126@126.com and Zunnan Huang; zn_huang@yahoo.com

Received 24 February 2016; Revised 3 June 2016; Accepted 3 July 2016

Academic Editor: Lei Yao

Copyright (C) 2016 Yong Liu et al. This is an open access article distributed under the Creative Commons Attribution License, which permits unrestricted use, distribution, and reproduction in any medium, provided the original work is properly cited.

Choline acetyltransferase (CHAT) rs3810950 and rs2177369 polymorphisms have been implicated in susceptibility to Alzheimer's disease (AD). Due to the inconsistent results from previous studies, a meta-analysis was performed to estimate the association between these polymorphisms and AD risk more precisely. Pooled results of our meta-analysis indicated CHAT rs 2177369 polymorphism was correlated with decreasing AD risk in one of five genetic models (dominant: OR = 0.77, 95\% CI: 0.62-0.96), while rs3810950 mutant was associated with $\mathrm{AD}$ development in three models (allelic: $\mathrm{OR}=1.18,95 \% \mathrm{CI}: 1.01-1.37$, homozygous: $\mathrm{OR}=1.63$, 95\% CI: $1.09-2.42$, and recessive: $\mathrm{OR}=1.65,95 \% \mathrm{CI}: 1.20-2.26$ ). In subgroup analysis by ethnicity, the association between CHAT rs3810950 polymorphism and AD risk was just found in the recessive model (OR $=1.47,95 \% \mathrm{CI}: 1.05-2.07)$ among Caucasians, while four genetic models (allelic: $\mathrm{OR}=1.23,95 \% \mathrm{CI}: 1.01-1.48$; homozygous: OR = 2.24, 95\% CI: 1.48-3.39; dominant: OR = 1.21, 95\% CI: 1.06-1.40; and recessive: $\mathrm{OR}=2.18,95 \%$ CI: $1.45-3.29$ ) assumed this association in Asians. In conclusion, our meta-analysis indicated CHAT rs2177369 polymorphism might play a protective role in $\mathrm{AD}$, while rs3810950 variant was a risk factor for $\mathrm{AD}$ but its single heterozygous mutations might not influence susceptibility to AD.

\section{Introduction}

Alzheimer's disease $(\mathrm{AD})$ is a neurodegenerative disorder characterized by severe damage of cognition. It is the most common form of age-related dementia [1]. Today, 35 million patients fight against dementia and most of them suffer from AD $[1,2]$. This brings huge losses to the social economy and seriously affects the long-term health-care system. Neuropathology of $\mathrm{AD}$ is characterized by the accumulation of extracellular $\beta$-amyloids $(\mathrm{A} \beta)$ in plaques and intracellular hyperphosphorylated tau protein [3]. Although the causes for the development of AD are still unclear, many studies showed that $\mathrm{AD}$ was triggered by multiple genetic and environmental factors $[2,4-6]$.

Genetic studies revealed that genetic factors played significant roles in the development of Alzheimer's disease [5]. Recently, several studies, including the large-scale genomewide association studies (GWAS) of $\mathrm{AD}$, have reported some 
susceptibility genes such as Apolipoprotein E (ApoE), Bridging Integrator 1 (BIN1), Clusterin (CLU), complement component receptor 1 (CR1), and choline O-acetyltransferase (CHAT) [7-10]. Among them, CHAT is the key enzyme responsible for the synthesis of a neurotransmitter acetylcholine and the target for many effective pharmacological therapies of $\mathrm{AD}$ [11].

CHAT gene has several genetic polymorphic loci such as rs1880676, rs2177369, rs868750, and rs3810950. In this paper, we focus only on the influence of the rs3810950 (G>A) and rs2177369 $(\mathrm{A}>\mathrm{G})$ polymorphisms on $\mathrm{AD}$ risk. We do not include other CHAT polymorphisms for this meta-analysis because all the other available polymorphisms do not meet the performance standard of conducting a mate-analysis due to their limited case-control studies.

The mutation of A/G in both rs3810950 and rs2177369 is fascinating due to its functionality. These polymorphisms in the CHAT gene may affect the synthesis of the enzyme, thereby amplifying the cholinergic neurotransmission deficits in AD [12]. The rs3810950 polymorphism has been proven to be associated with Alzheimer's disease in nine studies [10, 1219]. In these studies, the gene variation was found to associate with Alzheimer's disease among people in Asia, America, and five European countries. However, two other studies identified no association between CHAT rs3810950 and AD in British people $[20,21]$. Similarly, three previous articles, which investigated the relationship between rs2177369 and $\mathrm{AD}$ risk in the British and Italian population, respectively, also provided inconsistent results [21-23]. These controversial results from the earlier reports of different geographic areas might be caused by the relatively small size of each individual study and its low power to detect the true effect. We thus conducted a meta-analysis to give a more precise estimation of the association between these two CHAT polymorphisms and $\mathrm{AD}$ susceptibility.

\section{Methods and Materials}

2.1. Literature Search and Inclusion Criteria. Two investigators searched the PubMed, Embase, CNKI, Wanfang, and AlzGene databases to find all relevant records using the following keywords: "Choline acetyltransferase OR CHAT", "Alzheimer's disease OR AD" and "polymorphism OR polymorphisms OR variant OR mutation". The searches were last updated on May 15, 2016. Two authors took responsibility for literature searches to ensure the integrity of the data collection.

The inclusion criteria to select eligible articles in this meta-analysis were as follows: (1) the association of CHAT rs3810950 or rs2177369 polymorphism with Alzheimer's disease; (2) a case-control design; (3) complete genotype data including the number of homozygous mutant, heterozygous and wild genotypes to calculate ORs. On the other hand, the literatures were excluded if they met any of the following criteria: (1) abstracts, editorials, review articles, and unrelated meta-analyses; (2) studies without polymorphisms; (3) studies with other diseases or other polymorphisms; (4) studies with incomplete genotype data. Only one could be accepted if the publications were duplicated. Any disagreement regarding the inclusion of articles was resolved by discussion among the authors.

2.2. Data Extraction. One author extracted the following information from each study: (1) the first author's name; (2) the year of publication; (3) the country and ethnicity of the participants (patients and controls); (4) the number of Alzheimer's disease cases and controls; (5) the frequency of genotypes in AD cases and controls; (6) genotyping method; (7) diagnosis criteria of AD. Then, another author checked the data carefully to ensure they are complete and correct. In case-control studies, Hardy Weinberg Equilibrium (HWE) was used for quality assessment of genotype data. A highquality study was considered that its control group conformed to HWE. A study without HWE in controls was defined as a low quality one. Low quality studies were excluded in the sensitivity analysis. Newcastle-Ottawa Scale (NOS) criteria [24] were used to evaluate the quality of the case-control studies included in the meta-analysis. The evaluation of content in the NOS was classified into three independent aspects: object selection, comparability, and exposure assessment. In this retrospective analysis, an included study should get at least five points [25] in the NOS quality assessment.

2.3. Statistical Analysis. In this meta-analysis, the pooled odds ratios (ORs) with 95\% confidence intervals (CIs) were used to estimate the association of CHAT rs3810950 or rs2177369 polymorphisms with the risk of AD. We did not use environmental factors to adjust the poor ORs due to the very limited information provided in each of the included studies. We performed this meta-analysis using five genetic models: the allelic, homozygous, heterozygous, dominant, and recessive model. Chi-square test and $I^{2}$ test were used to calculate the heterogeneity of these genetic models. A $P$ value $<0.05$ and/or $I^{2}>50 \%$ indicated substantial heterogeneity, and then a random effect model was used; otherwise, a fixed effect model was used to calculate the ORs and 95\% CIs of any genetic model with unobserved heterogeneity [26, 27]. Additionally, meta-regression based on the covariates of sample size, ethnicity, and genotyping method was adopted to explore the source of heterogeneity. Subgroup analysis was also conducted according to ethnicity and genotyping method. At the same time, we did a stratified analysis by ApoE- $\varepsilon 4$ status.

Statistical power analysis was executed to estimate the suitability of the sample size employed to the power of the study. We assumed an unmatched case-control design and considered a two-sided $P$ value of 0.05 . "Venice criteria" [28] (Table S1, see Supplementary Material available online at http://dx.doi.org/10.1155/2016/9418163) were also applied to assess the credibility of the cumulative evidence of each meta-analyzed association under the genetic models we investigated. The evidence level is graded as strong, moderate, or weak.

Sensitivity analysis was applied to investigate the influence of the individual studies to the pooled results by omitting one study at a time. Both Begg's and Egger's tests were 


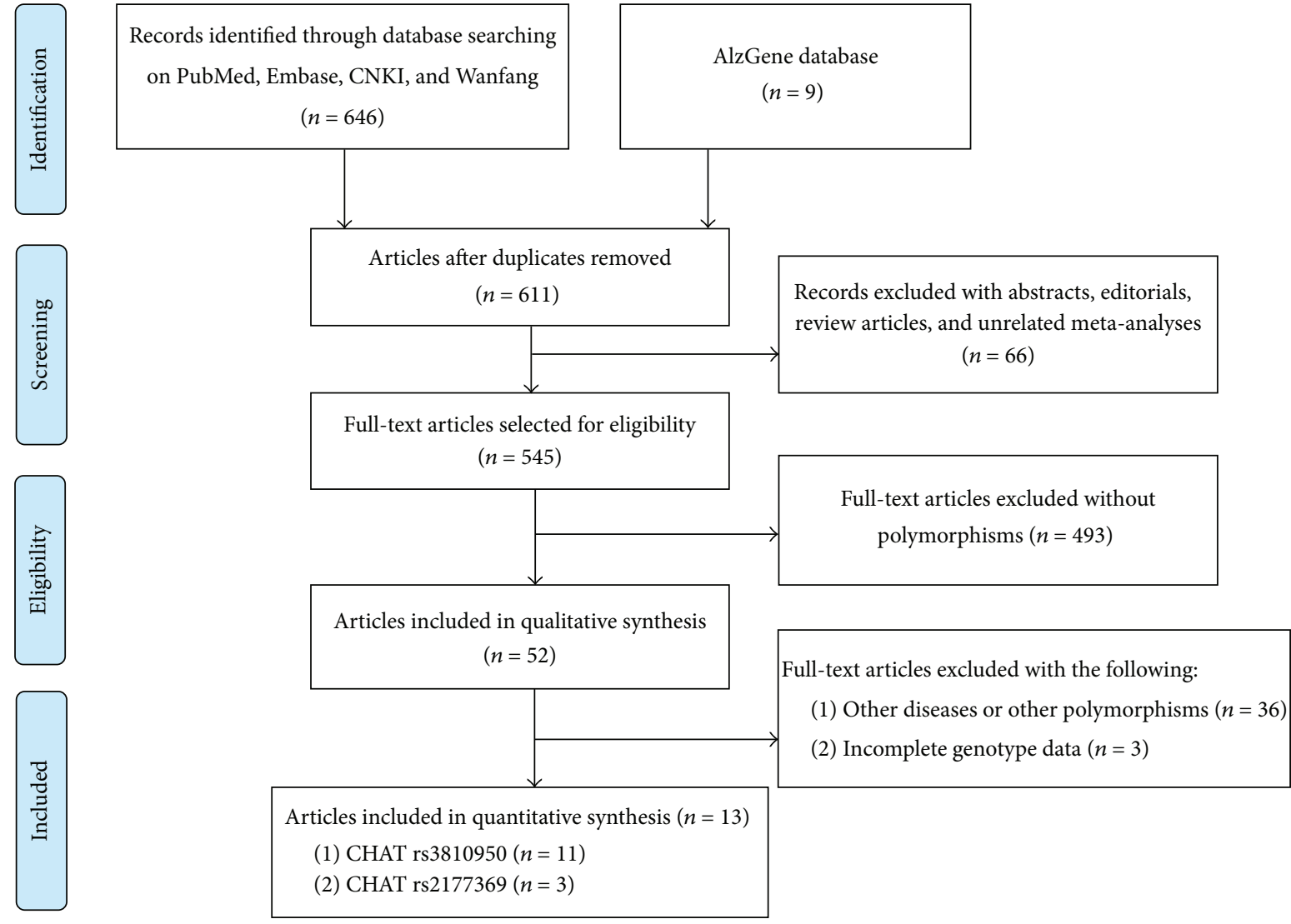

FIGURE 1: Flow diagram of the process used to select eligible studies.

used to assess publication bias and a $P$ value of less than 0.01 was considered statistically significant. The trim and fill method was also employed to identify and correct funnel plot asymmetry arising from publication bias. Data analysis was performed by professional software Review Manager 5.3 (Cochrane Informatics and Knowledge Management Department), STATA 14.0 (Stata Corporation College Station, Texas, USA), and Quanto software package (Version 1.2.4, http://biostats.usc.edu/software).

\section{Results}

3.1. Characteristics of the Studies. As shown in Figure 1, 655 related articles were first discovered from database searching up to May 15, 2016. Then, 611 articles were obtained after the duplicated publications were weeded out. Among them, 66 were abstracts, editorials, review articles, or unrelated metaanalyses, 493 lacked polymorphisms, and 39 related to other diseases and polymorphisms or had incomplete genotype data. Thus, we further discarded these studies (598) according to the exclusion criterions. Finally, we got thirteen eligible articles for our meta-analysis, which included 11 studies related to CHAT rs3810950 polymorphism and four studies linked to CHAT rs2177369 polymorphism. The baseline characteristics of all of these studies are listed in Table 1. The included studies conformed to HWE except for two: one reported by Mubumbila et al. [13] on CHAT rs3810950 polymorphism and the other one as Cook (1) study by Cook et al. [21] on CHAT rs2177369 polymorphism. Statistical power based on the given sample size of each study ranged from $5.00 \%$ to $90.29 \%$ under the dominant model and from $5.73 \%$ to $98.68 \%$ under the recessive model. The NOS results showed that the quality score of all the included studies satisfied the standard to reach five points or more (Table 2).

3.2. A Meta-Analysis of CHAT rs3810950 Polymorphism with $A D$ Risk. In this meta-analysis, a total of 11 studies [10, 1221] involving 3951 patients and 5963 controls were included to investigate the associations between CHAT rs3810950 and the risk of Alzheimer's disease (as shown in Table 1). Combined data showed that CHAT rs3810950 polymorphism was associated with an increased risk of $\mathrm{AD}$ in three of five genetic models (allelic A versus G: OR $=1.18,95 \%$ CI: $1.01-$ 1.37, $P=0.03$; homozygous AA versus GG: $\mathrm{OR}=1.63,95 \%$ CI: $1.09-2.42, P=0.02$; and recessive AA versus AG + GG: $\mathrm{OR}=1.65,95 \%$ CI: 1.20-2.26, $P<0.01$ ) (Figure 2) but no association was observed in the remaining two models (heterozygous AG versus GG: $\mathrm{OR}=0.99,95 \%$ CI: $0.90-$ $1.10, P=0.87$; dominant $\mathrm{AA}+\mathrm{AG}$ versus $\mathrm{GG}, \mathrm{OR}=1.08$, 95\% CI: $0.92-1.28, P=0.34$ ) (Table 3). Power analysis on the pooled sample size showed that the statistical power was $46.23 \%$ and $99.99 \%$, respectively, in the dominant and 


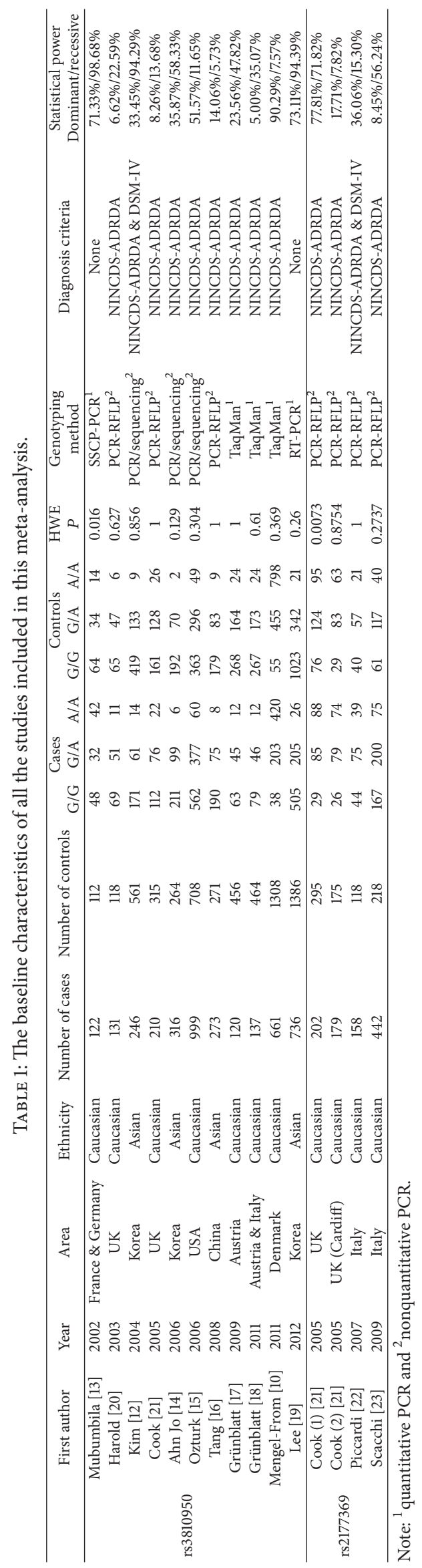


TABLE 2: Quality assessment scheme for the included literatures (Newcastle-Ottawa Scale).

\begin{tabular}{|c|c|c|c|c|c|c|c|c|c|c|}
\hline \multirow{2}{*}{ First author } & \multirow{2}{*}{ Year } & \multicolumn{4}{|c|}{ Selection } & \multirow{2}{*}{$\begin{array}{c}\text { Comparability } \\
\text { V }\end{array}$} & \multicolumn{3}{|c|}{ Exposure } & \multirow{2}{*}{ Total } \\
\hline & & I & II & III & IV & & VI & VII & VIII & \\
\hline Mubumbila [13] & 2002 & * & $*$ & & $*$ & $*$ & & $*$ & & $* * * * *$ \\
\hline Harold [20] & 2003 & * & * & & * & * & & $*$ & & $* * * * *$ \\
\hline Kim [12] & 2004 & $*$ & $*$ & $*$ & * & $* *$ & & $*$ & & $* * * * * * *$ \\
\hline Cook [21] & 2005 & * & * & & * & $*$ & & $*$ & & $* * * * *$ \\
\hline Ahn Jo [14] & 2006 & $*$ & * & $*$ & $*$ & $* *$ & & * & & $* * * * * * *$ \\
\hline Ozturk [15] & 2006 & * & * & & * & $* *$ & & * & & $* * * * * *$ \\
\hline Tang [16] & 2008 & * & * & & * & $* *$ & & * & & $* * * * * *$ \\
\hline Grünblatt [17] & 2009 & $*$ & $*$ & & $*$ & $*$ & & * & & $* * * * *$ \\
\hline Grünblatt [18] & 2011 & * & * & & * & * & & * & & $* * * * *$ \\
\hline Mengel-From [10] & 2011 & $*$ & $*$ & & $*$ & $* *$ & & $*$ & & $* * * * * *$ \\
\hline Lee $[19]$ & 2012 & * & * & * & * & $* *$ & & * & * & $* * * * * * * * *$ \\
\hline Piccardi [22] & 2007 & $*$ & $*$ & & $*$ & $*$ & & $*$ & & $* * * * *$ \\
\hline Scacchi [23] & 2009 & $*$ & $*$ & & * & $*$ & & * & & $* * * * *$ \\
\hline
\end{tabular}

Note: I: is the case definition adequate? II: representativeness of the cases. III: selection of controls. IV: definition of controls. V: comparability of cases and controls on the basis of the design or analysis. VI: ascertainment of exposure. VII: same method of ascertainment for cases and controls. VIII: nonresponse rate.

TABLE 3: Subgroup analyses of the association between CHAT rs3810950 polymorphism and Alzheimer's disease risk.

\begin{tabular}{|c|c|c|c|c|c|c|}
\hline & Genetic comparison & $I^{2}(\%)$ & Effect model & OR [95\% CI] & $P_{\mathrm{OR}}$ & Statistical power \\
\hline \multirow{5}{*}{ Overall } & A versus $G$ & 74 & Random & $1.18[1.01,1.37]$ & 0.03 & NA \\
\hline & AA versus GG & 72 & Random & $1.63[1.09,2.42]$ & 0.02 & NA \\
\hline & AG versus GG & 37 & Fixed & $0.99[0.90,1.10]$ & 0.87 & NA \\
\hline & $\mathrm{AA}+\mathrm{GA}$ versus $\mathrm{GG}$ & 62 & Random & $1.08[0.92,1.28]$ & 0.34 & $46.23 \%$ \\
\hline & AA versus GG + GA & 66 & Random & $1.65[1.20,2.26]$ & $<0.01$ & $99.99 \%$ \\
\hline \multicolumn{7}{|l|}{ Ethnicity-based } \\
\hline \multirow{5}{*}{ Caucasian (7) } & A versus G & 77 & Random & $1.16[0.94,1.42]$ & 0.16 & NA \\
\hline & AA versus GG & 74 & Random & $1.42[0.90,2.25]$ & 0.13 & NA \\
\hline & AG versus GG & 0 & Fixed & $0.88[0.77,1.00]$ & 0.06 & NA \\
\hline & $\mathrm{AA}+\mathrm{GA}$ versus $\mathrm{GG}$ & 60 & Random & $1.02[0.82,1.28]$ & 0.85 & $6.34 \%$ \\
\hline & AA versus GG + GA & 66 & Random & $1.47[1.05,2.07]$ & 0.03 & $99.99 \%$ \\
\hline \multicolumn{7}{|l|}{ Ethnicity-based } \\
\hline \multirow{5}{*}{ Asian (4) } & A versus $G$ & 52 & Random & $1.23[1.01,1.48]$ & 0.04 & NA \\
\hline & AA versus GG & 46 & Fixed & $2.24[1.48,3.39]$ & $<0.01$ & NA \\
\hline & AG versus GG & 5 & Fixed & $1.15[0.99,1.32]$ & 0.07 & NA \\
\hline & $\mathrm{AA}+\mathrm{GA}$ versus $\mathrm{GG}$ & 33 & Fixed & $1.21[1.06,1.40]$ & $<0.01$ & $77.90 \%$ \\
\hline & AA versus GG + GA & 40 & Fixed & $2.18[1.45,3.29]$ & $<0.01$ & $99.45 \%$ \\
\hline \multicolumn{7}{|l|}{ Genotyping-based } \\
\hline \multirow{5}{*}{ Quantitative PCR (5) } & A versus $G$ & 76.5 & Random & $1.32[1.05,1.65]$ & 0.02 & NA \\
\hline & AA versus GG & 80.6 & Random & $1.89[1.00,3.55]$ & 0.05 & NA \\
\hline & AG versus GG & 46.9 & Fixed & $1.08[0.93,1.26]$ & 0.32 & NA \\
\hline & $\mathrm{AA}+\mathrm{GA}$ versus GG & 64.3 & Random & $1.18[0.90,1.55]$ & 0.24 & $77.24 \%$ \\
\hline & AA versus GG + GA & 77.3 & Random & $1.94[1.18,3.19]$ & 0.01 & $99.99 \%$ \\
\hline \multicolumn{7}{|l|}{ Genotyping-based } \\
\hline \multirow{5}{*}{ Nonquantitative PCR (6) } & A versus $G$ & 62.8 & Random & $1.06[0.88,1.28]$ & 0.52 & NA \\
\hline & AA versus GG & 61.6 & Random & $1.40[0.82,2.37]$ & 0.22 & NA \\
\hline & AG versus GG & 18.8 & Fixed & $0.93[0.82,1.06]$ & 0.29 & NA \\
\hline & $\mathrm{AA}+\mathrm{GA}$ versus $\mathrm{GG}$ & 48.4 & Fixed & $1.01[0.83,1.21]$ & 0.95 & $5.11 \%$ \\
\hline & AA versus GG + GA & 55.7 & Random & $1.42[0.88,2.29]$ & 0.16 & $75.03 \%$ \\
\hline
\end{tabular}

Note: NA: not applicable. 


\begin{tabular}{|c|c|c|c|c|c|c|c|c|c|c|c|c|c|}
\hline \multirow{3}{*}{$\begin{array}{l}\text { Study or subgroup } \\
\text { Mubumbila et al. }\end{array}$} & \multicolumn{2}{|c|}{ Experimental } & \multicolumn{2}{|c|}{ Control } & \multirow{2}{*}{ Weight } & \multirow{2}{*}{$\begin{array}{c}\text { Odds ratio } \\
\mathrm{M}-\mathrm{H} \text {, random, 95\% CI }\end{array}$} & \multirow{2}{*}{\multicolumn{7}{|c|}{$\begin{array}{c}\text { Odds ratio } \\
\text { M-H, random, 95\% CI }\end{array}$}} \\
\hline & \multirow{2}{*}{$\begin{array}{c}\text { Events } \\
116\end{array}$} & \multirow{2}{*}{$\begin{array}{c}\text { Total } \\
244\end{array}$} & \multirow{2}{*}{$\begin{array}{c}\text { Events } \\
62\end{array}$} & \multirow{2}{*}{$\begin{array}{c}\text { Total } \\
224\end{array}$} & & & & & & & & & \\
\hline & & & & & $7.1 \%$ & $2.37[1.61,3.48]$ & & & & & $\longrightarrow$ & & \\
\hline Harold et al. & 73 & 262 & 59 & 236 & $6.8 \%$ & $1.16[0.78,1.73]$ & & & & & - & & \\
\hline Kim et al. & 89 & 492 & 151 & 1122 & $8.9 \%$ & $1.42[1.07,1.89]$ & & & & & 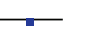 & & \\
\hline Cook et al. & 120 & 420 & 180 & 630 & $9.2 \%$ & $1.00[0.76,1.31]$ & & & & - & - & & \\
\hline Ahn et al. & 111 & 632 & 74 & 528 & $8.3 \%$ & $1.31[0.95,1.80]$ & & & & & 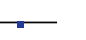 & & \\
\hline Ozturk et al. & 497 & 1998 & 394 & 1416 & $11.7 \%$ & $0.86[0.74,1.00]$ & & & & -1 & & & \\
\hline Tang et al. & 91 & 546 & 101 & 542 & $8.4 \%$ & $0.87[0.64,1.19]$ & & & & & & & \\
\hline Grünblatt et al. 2009 & 69 & 240 & 212 & 912 & $8.3 \%$ & $1.33[0.97,1.83]$ & & & & & 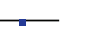 & & \\
\hline Grünblatt et al. 2011 & 70 & 274 & 221 & 928 & $8.5 \%$ & $1.10[0.80,1.50]$ & & & & & & & \\
\hline Mengel et al. & 1043 & 1322 & 2051 & 2616 & $11.5 \%$ & $1.03[0.88,1.21]$ & & & & $=$ & - & & \\
\hline Lee et al. & 257 & 1472 & 384 & 2772 & $11.3 \%$ & $1.32[1.11,1.56]$ & & & & & $\varpi$ & & \\
\hline Total (95\% CI) & & 7902 & & 11926 & $100.0 \%$ & $1.18[1.01,1.37]$ & & & & & & & \\
\hline Total events & 2536 & & 3889 & & & & $T$ & 1 & 1 & & 1 & 1 & $T$ \\
\hline Heterogeneity: $\tau^{2}=0$ & $\chi^{2}=37$. & $7, \mathrm{df}=$ & $0(P<0$. & $0001) ; I^{2}$ & $=74 \%$ & & 0.1 & 0.2 & 0.5 & 1 & 2 & 5 & 10 \\
\hline Test for overall effect: & $2.11(P$ & $0.03)$ & & & & & & ours [ & erimen & & Favo & ontrol] & \\
\hline
\end{tabular}

(a)

\begin{tabular}{|c|c|c|c|c|c|c|c|c|c|c|}
\hline \multirow{2}{*}{ Study or subgroup } & \multicolumn{2}{|c|}{ Experimental } & \multicolumn{2}{|c|}{ Control } & \multirow{2}{*}{ Weight } & \multirow{2}{*}{$\begin{array}{c}\text { Odds ratio } \\
\mathrm{M}-\mathrm{H} \text {, random, } 95 \% \mathrm{CI}\end{array}$} & \multirow{2}{*}{\multicolumn{4}{|c|}{$\begin{array}{c}\text { Odds ratio } \\
\text { M-H, random, 95\% CI }\end{array}$}} \\
\hline & \multirow{2}{*}{$\begin{array}{c}\text { Events } \\
42\end{array}$} & \multirow{2}{*}{$\begin{array}{c}\text { Total } \\
90\end{array}$} & \multirow{2}{*}{$\begin{array}{c}\text { Events } \\
14\end{array}$} & \multirow{2}{*}{$\begin{array}{c}\text { Total } \\
78\end{array}$} & & & & & & \\
\hline Mubumbila et al. & & & & & $9.5 \%$ & $4.00[1.96,8.15]$ & & & $\longrightarrow$ & \\
\hline Harold et al. & 11 & 80 & 6 & 71 & $7.0 \%$ & $1.73[0.60,4.94]$ & & - & 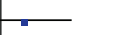 & \\
\hline Kim et al. & 14 & 185 & 9 & 428 & $8.4 \%$ & $3.81[1.62,8.97]$ & & & $\longrightarrow$ & \\
\hline Cook et al. & 22 & 134 & 26 & 187 & $10.3 \%$ & $1.22[0.66,2.25]$ & & & - & \\
\hline Ahn et al. & 6 & 217 & 2 & 194 & $4.2 \%$ & $2.73[0.54,13.69]$ & & - & & \\
\hline Ozturk et al. & 60 & 622 & 49 & 412 & $12.0 \%$ & $0.79[0.53,1.18]$ & & -7 & & \\
\hline Tang et al. & 8 & 198 & 9 & 188 & $7.5 \%$ & $0.84[0.32,2.22]$ & & 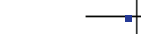 & - & \\
\hline Grünblatt et al. 2009 & 12 & 75 & 24 & 292 & $9.3 \%$ & $2.13[1.01,4.48]$ & & & - & \\
\hline Grünblatt et al. 2011 & 12 & 91 & 24 & 291 & $9.3 \%$ & $1.69[0.81,3.53]$ & & & $\square$ & \\
\hline Mengel et al. & 420 & 458 & 798 & 853 & $11.8 \%$ & $0.76[0.50,1.17]$ & & $\rightarrow-$ & & \\
\hline Lee et al. & 26 & 531 & 21 & 1044 & $10.6 \%$ & $2.51[1.40,4.50]$ & & & $\varpi$ & \\
\hline Total (95\% CI) & & 2681 & & 4038 & $100.0 \%$ & $1.63[1.09,2.42]$ & & & & \\
\hline Total events & 633 & & 982 & & & & & 1 & $T$ & \\
\hline Heterogeneity: $\tau^{2}=0$ & $.30 ; \chi^{2}=$ & 36.35 , & $\mathrm{df}=10$ & $P<0.00$ & 01); $I^{2}=$ & $72 \%$ & 0.001 & 0.1 & 10 & 1000 \\
\hline Test for overall effect: & $Z=2.40$ & $(P=0$ & & & & & & erimental] & Favou & \\
\hline
\end{tabular}

(b)

\begin{tabular}{|c|c|c|c|c|c|c|c|c|c|c|}
\hline \multirow{2}{*}{ Study or subgroup } & \multicolumn{2}{|c|}{ Experimental } & \multicolumn{2}{|c|}{ Control } & \multirow{2}{*}{ Weight } & \multirow{2}{*}{$\begin{array}{c}\text { Odds ratio } \\
\mathrm{M}-\mathrm{H} \text {, random, } 95 \% \mathrm{CI}\end{array}$} & \multirow{2}{*}{\multicolumn{4}{|c|}{$\begin{array}{c}\text { Odds ratio } \\
\mathrm{M}-\mathrm{H} \text {, random, } 95 \% \mathrm{CI}\end{array}$}} \\
\hline & \multirow{2}{*}{$\frac{\text { Events }}{42}$} & \multirow{2}{*}{$\begin{array}{c}\text { Total } \\
122\end{array}$} & \multirow{2}{*}{$\begin{array}{c}\text { Events } \\
14\end{array}$} & \multirow{2}{*}{$\begin{array}{c}\text { Total } \\
112\end{array}$} & & & & & & \\
\hline Mubumbila et al. & & & & & $9.4 \%$ & $3.67[1.87,7.20]$ & & & \multicolumn{2}{|l|}{ 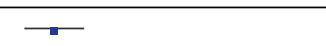 } \\
\hline Harold et al. & 11 & 131 & 6 & 118 & $6.0 \%$ & $1.71[0.61,4.78]$ & & & - & \\
\hline Kim et al. & 14 & 246 & 9 & 561 & $7.5 \%$ & $3.70[1.58,8.67]$ & & & $\longrightarrow$ & \\
\hline Cook et al. & 22 & 210 & 26 & 315 & $10.4 \%$ & $1.30[0.72,2.36]$ & & & \multirow[t]{2}{*}{-} & \\
\hline Ahn et al. & 6 & 316 & 2 & 264 & $3.1 \%$ & $2.54[0.51,12.67]$ & & & & \\
\hline Ozturk et al. & 60 & 999 & 49 & 708 & $13.2 \%$ & \multirow{2}{*}{$\begin{array}{l}0.86[0.58,1.27] \\
0.88[0.33,2.31]\end{array}$} & & & & \\
\hline Tang et al. & 8 & 273 & 9 & 271 & $6.5 \%$ & & & & & \\
\hline Grünblatt et al. 2009 & 12 & 120 & 24 & 456 & $8.8 \%$ & $2.00[0.97,4.13]$ & & & \multirow{2}{*}{5} & \\
\hline Grünblatt et al. 2011 & 12 & 137 & 24 & 464 & $8.9 \%$ & $1.76[0.86,3.62]$ & & & & \\
\hline Mengel et al. & 420 & 661 & 798 & 1308 & $15.6 \%$ & $1.11[0.92,1.35]$ & & & & \\
\hline Lee et al. & 26 & 736 & 21 & 1386 & $10.6 \%$ & $2.38[1.33,4.26]$ & & & $\longrightarrow$ & \\
\hline \multicolumn{2}{|l|}{ Total $(95 \%$ CI) } & 3951 & & 5963 & $100.0 \%$ & $1.65[1.20,2.26]$ & & & \multirow[t]{2}{*}{ 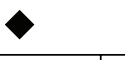 } & \\
\hline Total events & 633 & & 982 & & & & $\Gamma$ & 1 & & $\longrightarrow$ \\
\hline \multicolumn{7}{|c|}{ Heterogeneity: $\tau^{2}=0.16 ; \chi^{2}=29.48, \mathrm{df}=10(P=0.001) ; I^{2}=66 \%$} & 0.001 & 0.1 & 10 & 1000 \\
\hline \multicolumn{7}{|c|}{ Test for overall effect: $Z=3.12(P=0.002)$} & & erime & Favoul & \\
\hline
\end{tabular}

(c)

FIGURE 2: Forest plots of CHAT rs3810950 polymorphism and AD risk in three genetic models. (a) The allelic model (A versus G); (b) the homozygous model (AA versus GG); (c) the recessive model (AA versus AG + GG). 
recessive models. In general, our meta-analysis demonstrated that CHAT rs3810950 polymorphism increased the risk of Alzheimer's disease but only a heterozygous mutation of rs3810950 might not influence the susceptibility to AD.

3.3. Subgroup Analysis. The results from a subgroup analysis by ethnicity in Caucasians and Asians for all five genetic models are shown in Table 3. Only the recessive model (AA versus GG + GA: OR $=1.47,95 \%$ CI: $1.05-2.07, P=0.03$ ) established the association of CHAT rs3810950 polymorphism with AD risk among Caucasians, while four genetic models (allelic A versus $\mathrm{G}$ : $\mathrm{OR}=1.23$, 95\% CI: 1.01-1.48, $P=0.04$; homozygous AA versus $\mathrm{GG}$ : $\mathrm{OR}=2.24,95 \% \mathrm{CI}: 1.48-3.39, P<0.01$; dominant AA + AG versus GG, OR = 1.21, 95\% CI: 1.06-1.40, $P<0.01$; and recessive AA versus $\mathrm{GG}+\mathrm{GA}: \mathrm{OR}=2.18$, 95\% CI: $1.45-3.29, P<0.01)$ assumed this association among Asians. The statistical power of an Asian subgroup was $77.90 \%$ and $99.45 \%$ and that of a Caucasian subgroup was $6.34 \%$ and $99.99 \%$ calculated from power analysis, respectively, under the dominant and recessive models. Thus, only the homozygous mutant genotype AA might be related with the susceptibility to AD in a Caucasian population but only the heterozygous genotype AG might not be linked to increasing $\mathrm{AD}$ risk in an Asian population. In addition, a further analysis of studies involving British only indicated that no association between CHAT rs3810950 polymorphism and Alzheimer's disease was found in British people for all five genetic models (Table S2 in Supporting Information). The statistical power calculated for the British group was $5.41 \%$ and $26.56 \%$ under the dominant and recessive models, respectively.

In the subgroup analysis according to the genotyping method [29], conflict results were obtained based on two different subgroups known as quantitative PCR and nonquantitative PCR (Table 3). The pooled ORs calculated for the quantitative PCR group showed that CHAT rs3810950 polymorphism contributed to increasing $\mathrm{AD}$ risk in three of five genetic models (allelic A versus G: OR $=1.32$, 95\% CI: 1.05-1.65, $P=0.02$; homozygous AA versus GG: $\mathrm{OR}=$ 1.89, 95\% CI: $1.00-3.55, P=0.05$; and recessive AA versus GG + GA: OR $=1.94,95 \%$ CI: $1.18-3.19, P=0.01)$ while no association was found in all the five genetic models for the nonquantitative PCR group (Table 3). The statistical power of a quantitative PCR subgroup was $77.24 \%$ and $99.99 \%$ and that of a nonquantitative PCR subgroup was $5.11 \%$ and $75.03 \%$, respectively, under the dominant and recessive models.

3.4. Stratified Analysis. Because four included studies in this meta-analysis provided the genotype data of CHAT rs3810950 polymorphism and ApoE- $\varepsilon 4$ allele [10, 12, 14, 15], we further operated a risk-stratification analysis to calculate the association of rs3810950 polymorphism with AD based on the absence and presence of ApoE- $\varepsilon 4$. The combined influence of CHAT rs3810950 polymorphism and ApoE- $\varepsilon 4$ allele on Alzheimer's disease is shown in Table 4. For all the comparisons, the GG + GA genotype within non-ApoE$\varepsilon 4$ carriers served as a reference. Among ApoE- $\varepsilon 4$ carriers, individuals with the GG + GA genotype showed a significantly increased risk of Alzheimer's disease (OR $=3.46,95 \%$ CI: $1.78-6.71, P<0.001)$. A substantial interaction was also found between ApoE- $\varepsilon 4$ carriers and the AA genotype (OR $=4.87,95 \% \mathrm{CI}: 1.67-14.22, P=0.004)$. All of these evidences manifested that ApoE- $\varepsilon 4$ allele could be a vital factor in the Alzheimer's disease caused by CHAT rs3810950 polymorphism. Under the existence of ApoE- $\varepsilon 4$, the risk of Alzheimer's disease increased notably when the genotype of rs3810950 was GG + GA or AA.

3.5. Strength of the Evidence. When Venice criteria were applied to assess credibility, results under all five genetic models were graded as "A" for "amount of evidence" of all meta-analyses except " $B$ " for British subgroup analysis, "A," "B," or "C" for "replication consistency," and " $A$ " for "protection from bias" of the overall analysis (Table S3). These results suggested that there was moderate or weak evidence of the association between rs3810950 polymorphism and AD susceptibility.

3.6. A Meta-Analysis between CHAT rs2177369 Polymorphism and $A D$ Risk. A meta-analysis of the association between CHAT rs2177369 polymorphism and AD risk included four independent studies [21-23] with a total of 981 cases and 806 controls (as shown in Table 1). Combined data revealed that CHAT rs2177369 polymorphism was correlated with a decreased risk of $A D$ in the dominant model $(G G+G A$ versus AA: $\mathrm{OR}=0.77,95 \% \mathrm{CI}: 0.62-0.96, P=0.02$, statistical power $=69.05 \%)$ but no connection was detected in the rest four genetic models (allelic $\mathrm{G}$ versus $\mathrm{A}$ : $\mathrm{OR}=0.85,95 \% \mathrm{CI}$ : $0.61-1.18, P=0.34$; homozygous $\mathrm{GG}$ versus $\mathrm{AA}: \mathrm{OR}=0.73$, 95\% CI: $0.40-1.32, P=0.30$; heterozygous GA versus AA: $\mathrm{OR}=0.80,95 \% \mathrm{CI}: 0.63-1.01, P=0.06$; recessive $\mathrm{GG}$ versus $\mathrm{GA}+\mathrm{AA}: \mathrm{OR}=0.85,95 \% \mathrm{CI}: 0.49-1.47, P=0.55$, statistical power $=30.29 \%)($ Table 5, Figure 3$)$. Thus, the overall analysis indicated that CHAT rs2177369 polymorphism could reduce the risk of $\mathrm{AD}$ but the association might be only slightly correlated.

We also performed two subanalyses which excluded Cook (1) and Scacchi studies, respectively, because these two studies provided entirely opposite effects of CHAT rs2177369 polymorphism on $\mathrm{AD}$ risk [21, 23]. The meta-analysis from the exclusion of Cook (1) study showed that CHAT rs2177369 mutant was not associated with $\mathrm{AD}$ risk in all five genetic models (allelic G versus A: OR $=0.96,95 \%$ CI: $0.70-1.31$, $P=0.78$; homozygous $\mathrm{GG}$ versus $\mathrm{AA}: \mathrm{OR}=0.91,95 \% \mathrm{CI}$ : $0.52-1.59, P=0.73$; heterozygous $\mathrm{GA}$ versus $\mathrm{AA}: \mathrm{OR}=0.83$, 95\% CI: $0.62-1.10, P=0.19$; dominant $\mathrm{GG}+\mathrm{GA}$ versus $\mathrm{AA}$ : $\mathrm{OR}=0.87,95 \% \mathrm{CI}: 0.67-1.14, P=0.32$, statistical power $=$ 19.62\%; recessive GG versus GA + AA: OR $=1.04,95 \% \mathrm{CI}$ : $0.63-1.71, P=0.88$, statistical power $=5.97 \%)($ Table 5, Figure $\mathrm{S} 1)$. On the other hand, the meta-analysis from the exclusion of Scacchi study showed that CHAT rs2177369 variant was statistically significant associated with decreasing AD risk in all five genetic models (allelic $\mathrm{G}$ versus $\mathrm{A}$ : $\mathrm{OR}=0.73,95 \% \mathrm{CI}$ : $0.61-0.86, P<0.01$; homozygous GG versus AA: $\mathrm{OR}=0.54$, 95\% CI: 0.39-0.76, $P<0.01$; heterozygous GA versus AA: $\mathrm{OR}=0.76,95 \% \mathrm{CI}: 0.58-0.99, P=0.05$; dominant GG + GA versus AA: $\mathrm{OR}=0.68,95 \% \mathrm{CI}: 0.53-0.88, P<0.01$, statistical power $=86.83 \%$; recessive $\mathrm{GG}$ versus $\mathrm{GA}+\mathrm{AA}: \mathrm{OR}=0.65$, 95\% CI: $0.48-0.87, P<0.01$, statistical power $=73.79 \%$ ) 


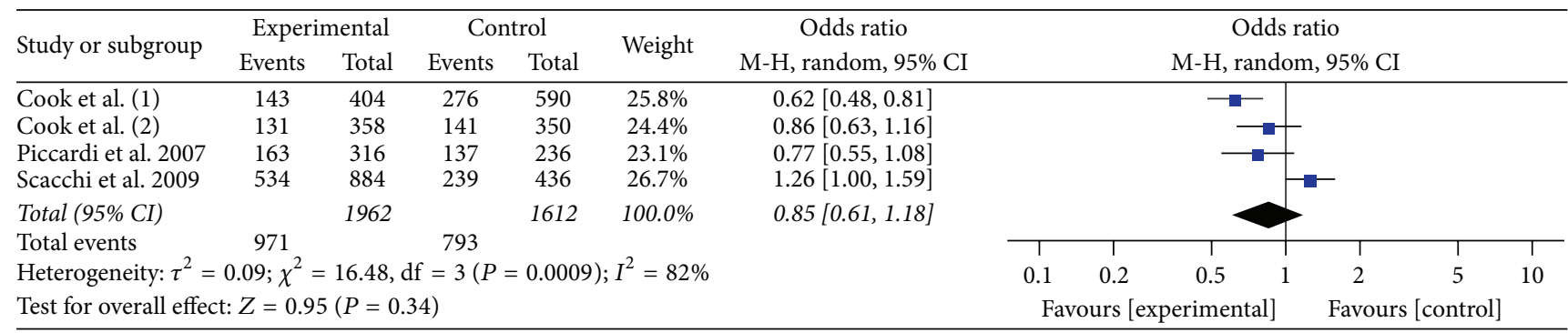

(a)

\begin{tabular}{|c|c|c|c|c|c|c|c|c|c|c|c|c|}
\hline \multirow{2}{*}{ Study or subgroup } & \multicolumn{2}{|c|}{ Experimental } & \multicolumn{2}{|c|}{ Control } & \multirow{2}{*}{ Weight } & \multirow{2}{*}{$\begin{array}{c}\text { Odds ratio } \\
\mathrm{M}-\mathrm{H} \text {, random, } 95 \% \mathrm{CI}\end{array}$} & \multirow{2}{*}{\multicolumn{6}{|c|}{$\begin{array}{c}\text { Odds ratio } \\
\mathrm{M}-\mathrm{H} \text {, random, } 95 \% \mathrm{CI}\end{array}$}} \\
\hline & \multirow{2}{*}{$\frac{\text { Events }}{29}$} & Total & Events & \multirow{2}{*}{ Total } & & & & & & & & \\
\hline Cook et al. (1) & & 117 & 76 & & $26.2 \%$ & $0.41[0.25,0.69]$ & & & - & & & \\
\hline Cook et al. (2) & 26 & 100 & 29 & 92 & $24.0 \%$ & $0.76[0.41,1.43]$ & & & & & & \\
\hline Piccardi et al. 2007 & 44 & 83 & 40 & 61 & $22.9 \%$ & $0.59[0.30,1.17]$ & & & - & - & & \\
\hline Scacchi et al. 2009 & 167 & 242 & 61 & 101 & $26.9 \%$ & $1.46[0.90,2.37]$ & & & & & & \\
\hline Total $(95 \%$ CI) & & 542 & & 425 & $100.0 \%$ & $0.73[0.40,1.32]$ & & & & & & \\
\hline \multirow{3}{*}{\multicolumn{7}{|c|}{$\begin{array}{l}\text { Total events } 266 \quad 206 \\
\text { Heterogeneity: } \tau^{2}=0.28 ; \chi^{2}=12.94, \mathrm{df}=3(P=0.005) ; I^{2}=77 \% \\
\text { Test for overall effect: } Z=1.04(P=0.30)\end{array}$}} & & & & & & \\
\hline & & & & & & & 0.1 & 0.2 & 0.5 & 2 & 5 & 10 \\
\hline & & \multicolumn{5}{|c|}{ Test for overall effect: $Z=1.04(P=0.30)$} & \multicolumn{6}{|c|}{ Favours [experimental] $\quad$ Favours [control] } \\
\hline
\end{tabular}

(b)

\begin{tabular}{|c|c|c|c|c|c|c|c|c|c|c|c|c|}
\hline \multirow{2}{*}{ Study or subgroup } & \multicolumn{2}{|c|}{ Experimental } & \multicolumn{2}{|c|}{ Control } & \multirow{2}{*}{ Weight } & Odds ratio & \multirow{2}{*}{\multicolumn{6}{|c|}{$\begin{array}{c}\text { Odds ratio } \\
\text { M-H, fixed, } 95 \% \text { CI }\end{array}$}} \\
\hline & Events & Total & Events & Total & & M-H, fixed, 95\% CI & & & & & & \\
\hline Cook et al. (1) & 85 & 173 & 124 & 219 & $34.7 \%$ & $0.74[0.50,1.10]$ & \multicolumn{6}{|c|}{$\longrightarrow+$} \\
\hline Cook et al. (2) & 79 & 153 & 83 & 146 & $25.6 \%$ & $0.81[0.51,1.28]$ & \multirow{2}{*}{\multicolumn{3}{|c|}{$\longrightarrow$}} & - & & \\
\hline Piccardi et al. 2007 & 75 & 114 & 57 & 78 & $14.4 \%$ & $0.71[0.38,1.33]$ & & & & & & \\
\hline Scacchi et al. 2009 & 200 & 275 & 117 & 157 & $25.3 \%$ & $0.91[0.58,1.42]$ & \multicolumn{3}{|r|}{$\longrightarrow$} & & & \\
\hline \multicolumn{2}{|c|}{ Total $(95 \%$ CI) } & \multirow{2}{*}{715} & & 600 & $100.0 \%$ & $0.80[0.63,1.01]$ & \multirow{2}{*}{\multicolumn{3}{|c|}{ 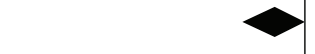 }} & & & \\
\hline \multirow{3}{*}{\multicolumn{7}{|c|}{$\begin{array}{l}\text { Total events } \quad 439 \quad 381 \\
\text { Heterogeneity: } \chi^{2}=0.62, \mathrm{df}=3(P=0.89) ; I^{2}=0 \% \\
\text { Test for overall effect: } Z=1.92(P=0.06)\end{array}$}} & & & & \multirow{3}{*}{\multicolumn{2}{|c|}{$\begin{array}{cc} & \\
2 & 5 \\
\text { Favours } & \text { [control] }\end{array}$}} & \\
\hline & & & & & & & 0.1 & & \multirow{2}{*}{0.51} & & & 10 \\
\hline & & & & & & & \multicolumn{2}{|c|}{ Favours [experimental] } & & & & \\
\hline
\end{tabular}

(c)

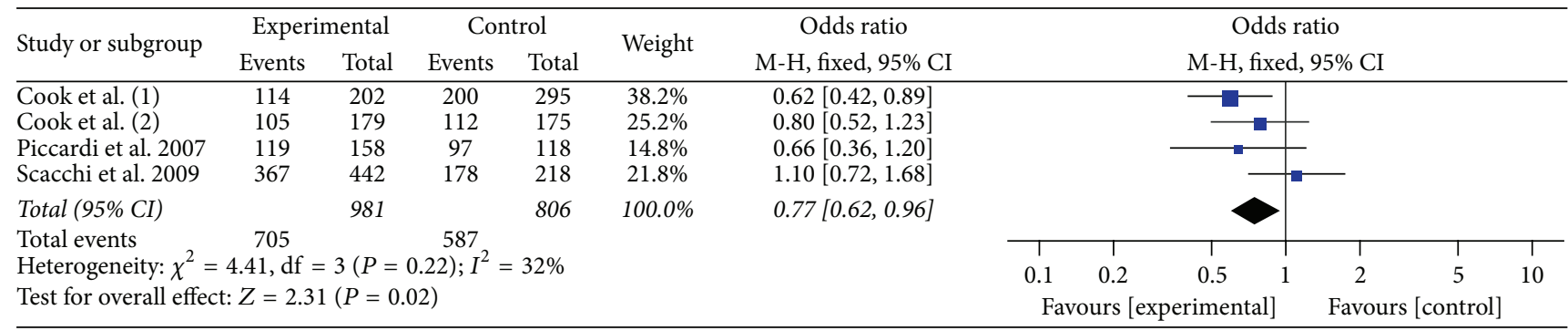

(d)

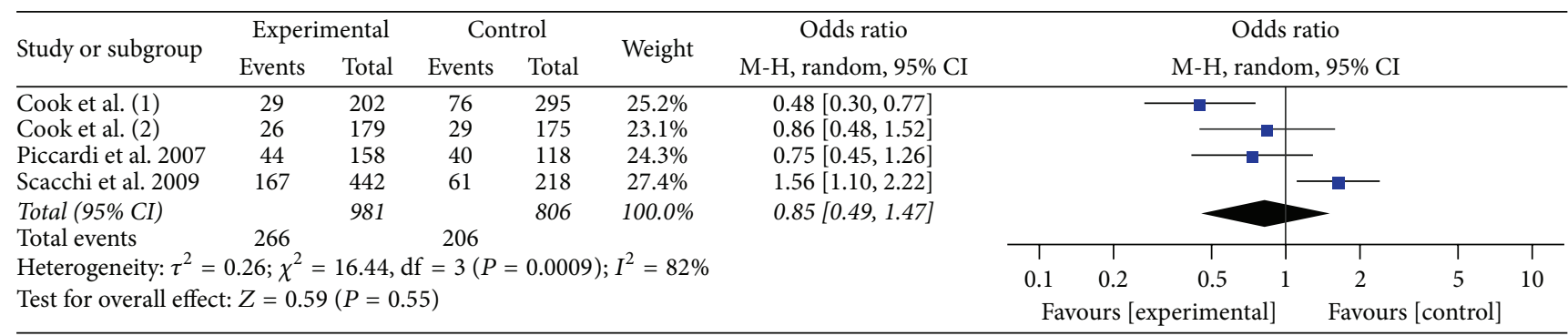

(e)

FIGURE 3: Forest plots of CHAT rs2177369 polymorphism and AD risk in five genetic models. (a) The allelic model (G versus A); (b) the homozygous model (GG versus AA); (c) the heterozygous model (GA versus AA); (d) the dominant model (GG + GA versus AA); (e) the recessive model (GG versus $\mathrm{GA}+\mathrm{AA}$ ). 
TABLE 4: Risk of Alzheimer's disease associated with CHAT rs3810950 polymorphism by ApoE- $\varepsilon 4$ status.

\begin{tabular}{|c|c|c|c|c|c|c|c|c|}
\hline \multirow{2}{*}{ Genetic comparison } & \multicolumn{4}{|c|}{ Non-ApoE- $\varepsilon 4$ carriers } & \multicolumn{4}{|c|}{ ApoE- $\varepsilon 4$ carriers } \\
\hline & Cases & Controls & OR $(95 \% \mathrm{CI})$ & $P$ & Cases & Controls & OR $(95 \%$ CI $)$ & $P$ \\
\hline $\mathrm{GG}+\mathrm{GA}$ & 851 & 1605 & 1 (reference) & NA & 862 & 377 & $3.46(1.78-6.71)$ & $<0.001$ \\
\hline $\mathrm{AA}$ & 292 & 673 & $1.03(0.62-1.71)$ & 0.08 & 203 & 185 & $4.87(1.67-14.22)$ & 0.004 \\
\hline
\end{tabular}

Note: NA: not applicable.

TABLE 5: Meta-analysis of the association between CHAT rs2177369 polymorphism and Alzheimer's disease risk.

\begin{tabular}{|c|c|c|c|c|c|c|}
\hline Meta-analysis & Genetic comparison & $I^{2}(\%)$ & Effect model & OR [95\% CI] & $P_{\mathrm{OR}}$ & Statistical power \\
\hline \multirow{5}{*}{ Overall } & G versus $A$ & 82 & Random & $0.85[0.61,1.18]$ & 0.34 & NA \\
\hline & GG versus AA & 77 & Random & $0.73[0.40,1.32]$ & 0.3 & NA \\
\hline & GA versus AA & 0 & Fixed & $0.80[0.63,1.01]$ & 0.06 & NA \\
\hline & $\mathrm{GG}+\mathrm{GA}$ versus $\mathrm{AA}$ & 32 & Fixed & $0.77[0.62,0.96]$ & 0.02 & $69.05 \%$ \\
\hline & GG versus $\mathrm{AA}+\mathrm{GA}$ & 82 & Random & $0.85[0.49,1.47]$ & 0.55 & $30.29 \%$ \\
\hline \multirow{5}{*}{ Analysis without Cook (1) study } & G versus $A$ & 72 & Random & $0.96[0.70,1.31]$ & 0.78 & NA \\
\hline & GG versus AA & 62 & Random & $0.91[0.52,1.59]$ & 0.73 & NA \\
\hline & GA versus AA & 0 & Fixed & $0.83[0.62,1.10]$ & 0.19 & NA \\
\hline & $\mathrm{GG}+\mathrm{GA}$ versus $\mathrm{AA}$ & 7 & Fixed & $0.87[0.67,1.14]$ & 0.32 & $19.62 \%$ \\
\hline & GG versus AA + GA & 69 & Random & $1.04[0.63,1.71]$ & 0.88 & $5.97 \%$ \\
\hline \multirow{5}{*}{ Analysis without Scacchi study } & G versus $A$ & 22 & Fixed & $0.73[0.61,0.86]$ & $<0.01$ & NA \\
\hline & GG versus AA & 13 & Fixed & $0.54[0.39,0.76]$ & $<0.01$ & NA \\
\hline & GA versus AA & 0 & Fixed & $0.76[0.58,0.99]$ & 0.05 & NA \\
\hline & GG + GA versus AA & 0 & Fixed & $0.68[0.53,0.88]$ & $<0.01$ & $86.83 \%$ \\
\hline & GG versus $\mathrm{AA}+\mathrm{GA}$ & 26 & Fixed & $0.65[0.48,0.87]$ & $<0.01$ & $73.79 \%$ \\
\hline
\end{tabular}

Note: NA: not applicable.

(Table 5, Figure S2). Thus, the sub-analysis of the exclusion of Cook (1) study showed that CHAT rs2177369 polymorphism did not affect AD risk while that from the exclusion of Scacchi study indicated that this polymorphism played a protective role in the Alzheimer's disease.

3.7. Heterogeneity, Meta-Regression, Sensitivity Analysis, and Publication Bias. Our meta-analysis showed evidence of genetic heterogeneity in all the genetic models of the two polymorphisms except for CHAT rs2177369 polymorphism in the heterozygous model $\left(I^{2}=0 \%\right.$, as shown in Tables 3 and 5). Through the calculation of between-study heterogeneity in clinical AD samples, we found significant heterogeneity among studies on CHAT rs3810950 polymorphism in the allelic model $\left(I^{2}=74 \%\right)$, homozygous model $\left(I^{2}=72 \%\right)$, dominant model $\left(I^{2}=62 \%\right)$, and recessive model $\left(I^{2}=66 \%\right)$ and on CHAT rs2177369 polymorphism in the allelic model $\left(I^{2}=82 \%\right)$, homozygous model $\left(I^{2}=77 \%\right)$, and recessive model $\left(I^{2}=82 \%\right)$. Meanwhile, moderate heterogeneity was observed in the remaining two models as the heterozygous model $\left(I^{2}=37 \%\right)$ on CHAT rs3810950 polymorphism and the dominant model $\left(I^{2}=32 \%\right)$ on CHAT rs2177369 polymorphism. On CHAT rs3810950 polymorphism, the results of the meta-regression showed that the sample size, ethnicity, and genotyping method did not contribute to the heterogeneity of genetic models (data not shown here). Sensitivity analysis was then performed to assess the impact of the independent studies which caused obvious heterogeneity in those four models on CHAT rs3810950 polymorphism. We explored the influence of these studies on the pooled OR by removing each one at a time and found no significant change of our metaanalysis results. We did not perform both meta-regression and sensitivity analysis on CHAT rs2177369 polymorphism due to only four case-control studies involved.

We further performed Begg's and Egger's tests to assess publication bias in the study of the genetic association between CHAT rs3810950 polymorphism and AD risk. As shown in Table S4, no obvious publication bias was detected according to the obtained $P$ values for these genetic models. In addition, we did not observe any obvious asymmetry from the shape of Begg's funnel plot (Figure S3). In general, the effect of publication bias could be negligible in the included studies on CHAT rs3810950 polymorphism. However, we estimated the risk of publication bias neither in the subgroup analyses nor in the meta-analysis on CHAT rs2177369 polymorphism because the number of case-control studies in these studies was less than ten.

\section{Discussion}

The association of CHAT rs3810950 polymorphism with Alzheimer's disease was previously reported in one published meta-analysis [30]. However, the earlier work only included three case-control studies with 1183 incident AD patients and 1705 controls. In order to give a more precise estimation of the association between CHAT rs3810950 polymorphism and $\mathrm{AD}$, we collected 11 eligible studies with a total of 3951 cases and 5963 controls to perform this meta-analysis. Based on the enriched data we got, five genetic models were carried 
out to further clarify the impact of rs3810950 variant on AD. As a result, our meta-analysis showed that CHAT rs3810950 polymorphism was associated with the risk of Alzheimer's disease but such an association might not exist for only single-allele mutant in the general population (Figure 2 and Table 3). In other words, people with the homozygous mutant genotype (AA) should have a much higher risk of developing Alzheimer's disease than those with the heterozygous (AG) and wide-type genotype (GG). The results could be quite reliable providing that the pooled OR showed statistical significance and the statistical power was nearly $100 \%$ under the recessive model. These more refined findings in our study could be useful for future genetic studies on AD.

In order to get more detailed information about this association, we further carried out subgroup analyses by ethnicity and by genotyping method, respectively. On one hand, subgroup analysis by ethnicity indicated that Caucasians might have a lower risk of Alzheimer's disease than Asians (Table 3). In addition, among Caucasians, the British might have much less possibility of suffering from Alzheimer's disease even if their CHAT rs3810950 genotype was AA when compared to the people from other geographic regions (Table 3 and Table S2). Nevertheless, there were only two British studies [20, 21]. This might result in false negative findings and thus people should be more cautious about this result. Low statistical power calculated for the British group clearly supported this point (Table S2). On the other hand, subgroup analysis by genotyping method implied that the genotyping error or bias might exist in our meta-analysis due to the inconsistent results obtained for the quantitative PCR and nonquantitative PCR subgroups, respectively. Besides, the high and low statistical power calculated, respectively, for the quantitative PCR and nonquantitative PCR subgroups could indicate that no association was observed between CHAT rs3810950 polymorphism and AD risk for the nonquantitative PCR subgroup probably just due to its small sample size effect (Table 3 ).

A further stratified analysis of the association between CHAT rs3810950 allele and Alzheimer's disease according to the ApoE- $\varepsilon 4$ status showed that, with the same rs3810950 polymorphism, ApoE- $\varepsilon 4$ carriers exhibited a significantly higher incidence of $\mathrm{AD}$ than the non-ApoE- $\varepsilon 4$ carriers (Table 4). Thus, interaction between CHAT rs3810950 polymorphism and ApoE- $\varepsilon 4$ allele could be a huge risk factor for Alzheimer's disease. However, only four studies were included in this risk-stratification analysis and we should also treat this result with caution.

Up to date, four independent studies have investigated the link between CHAT rs2177369 polymorphism and the risk of AD. Among them, two studies [21, 22] indicated no relationship between this polymorphism and the susceptibility to the disease. The other two studies [21, 23] found the association between CHAT rs2177369 variant and AD risk but provided the opposite results. Cook et al. [21] denoted that carrying the minor alleles (GG + GA) was significantly protective with respect to carrying the homozygous widetype allele (AA), while Scacchi et al. [23] showed that the homozygous mutant (GG) was a risk factor compared with the GA + AA genotypes. Our meta-analysis results indicated that the rs2177369 polymorphism played a protective role in the disease, which agreed well with Cook (1) study. Though the results from the overall analysis based on the four independent studies only supported a weak association, the subanalysis of the exclusion of Scacchi study clearly suggested that this polymorphism was a protective factor for $\mathrm{AD}$ (Table 5). On the other hand, we considered that the results of no association between CHAT rs2177369 polymorphism and $\mathrm{AD}$ risk derived from the subanalysis excluding the Cook (1) study were unreliable since they did not reach the statistical significance and the statistical power was quite low (19.62\% for dominant model and $5.97 \%$ for recessive model, Table 5). In addition, the substantial heterogeneity existed among the included studies under the allelic, homozygous, and recessive models (Table 5). However, additional studies with larger sample sizes need to be further performed for verifying the potential protective association between CHAT rs2177369 polymorphism and the risk of Alzheimer's disease.

Presence of heterogeneity was detected in this retrospective study. It was known that age, gender, ethnicity, lifestyle habits (smoking and alcohol), education, vascular risk, ApoE$\varepsilon 4$ status, and other genetic or environmental factors influence AD onset [31-34]. Therefore, we have reasons to believe that these potential causes may account for the heterogeneity and the different results among the included studies. We tried to extract the data of these $\mathrm{AD}$ risk factors for further analysis. However, we could not conduct the subgroup analysis by age, gender, vascular risk, education or habits, and so forth due to their insufficient data. For CHAT rs3810950 polymorphism with AD risk, we could not clarify the sources of significant between-study heterogeneity neither from the ethnicity or genotyping method based subgroup analyses and ApoE-ع4-based stratified analysis nor from the metaregression according to the variables of sample size, ethnicity, and genotyping method. Therefore, other factors such as age, habits, and education may cause the high heterogeneity among studies on rs3810950 polymorphism. For CHAT rs2177369 polymorphism with $\mathrm{AD}$ risk, however, we found that substantial heterogeneity only existed between the study investigated by Scacchi et al. [23] and others performed by Cook et al. [21] and Piccardi et al. [22], respectively. When excluding the Scacchi study, only unimportant betweenstudy heterogeneity was observed in the meta-analysis $\left(I^{2} \leq\right.$ $26 \%$ for all five genetic models, Table 5 and Figure S2). Thus, we also considered that the potential factor such as age, education, habits, or even treatment with anti-inflammatory drugs in the Scacci study, and so forth may account for the high heterogeneity between this study and others on rs2177369 polymorphism.

Our meta-analysis had several advantages. Firstly, this study is the first meta-analysis to investigate the association of CHAT rs2177369 polymorphism with the development of Alzheimer's disease. In addition, though a previous meta-analysis [30] has explored the relationship of CHAT rs3810950 variant with the susceptibility to $A D$, our study was performed based on a much larger sample size. Secondly, five genetic models were used in this meta-analysis. As a result, 
our study not only demonstrated the association between these two polymorphisms with $\mathrm{AD}$, but also clarified that the homozygous and heterozygous mutant genotypes might play the potentially different roles in AD susceptibility. Thirdly, subgroup analyses, subanalyses, and meta-regression were conducted to explore the source of heterogeneity under the genetic models in this retrospective analysis. Ethnicitybased subgroup analysis also helped us to investigate the possibly different impact of CHAT rs3810950 polymorphism on $\mathrm{AD}$ risk in different ethnic groups. Fourthly, a riskstratification analysis of the association by ApoE- $\varepsilon 4$ status was also carried out in our study. This analysis assisted us to detect the promising effect of gene-gene interaction on the development of disease. Fifthly, we not only used Begg's and Egger's tests to assess the risk of publication bias but also employed the trim and fill method to identify and correct funnel plot asymmetry arising from publication bias. Sixthly, the NOS criteria were performed to evaluate the quality of the included studies and Venice criteria were applied to assess the cumulative evidence of the associations in our meta-analysis. Finally, statistical power analysis was also executed to estimate the effect of the sample size on the power of the study and this increased the credibility of our result.

Some limitations should be also recognized in this metaanalysis. Firstly, a subgroup analysis based on age, gender, or lifestyle habits may also contribute to the association of CHAT rs3810950 and rs2177369 polymorphisms with Alzheimer's disease $[35,36]$, but we did not perform such an extensive analysis because of the limited data. Secondly, the geographic regions of the participants were restricted. We could not find a study on CHAT rs3810950 loci to investigate the population in Africa, Australia, or South America, while studies on CHAT rs2177369 loci are only involved in the British and Italian populations. This limitation might lead our results into less accuracy. Thirdly, we could not use environmental factors to adjust the pooled ORs of the association between the genetic polymorphism and the disease because of the unavailability of environmental information in the included studies. Finally, an obvious heterogeneity was observed in this meta-analysis. The study designs, populations (age and gender), habits, and geographical location may contribute to the heterogeneity.

Alzheimer's disease is the most prevalent neurodegenerative disease in the elderly and it has caused serious damage to our health $[1,2,6]$. Substantial progress has been made towards characterization of Alzheimer's disease, but presently there are still no efficient therapies for Alzheimer's disease, and the pathogenic mechanism of Alzheimer's disease still remains unclear $[4,37,38]$. Thus, it is urgent to enrich our understanding of $\mathrm{AD}$ pathogenesis or we will almost surely fail to develop effective treatments for Alzheimer's disease. The results from this meta-analysis would help us to reach this goal.

\section{Competing Interests}

The authors declare that there is no conflict of interests regarding the publication of this paper.

\section{Authors' Contributions}

Yong Liu and Qicong Chen are equal contributors.

\section{Acknowledgments}

This work was supported by National Natural Science Foundation of China (31170676), Traditional Chinese Medicine Bureau Foundation of Guangdong Province, China (20151264), and the funds from Sail Plan "the Introduction of the Shortage of Top-Notch Talent” Project (YueRenCaiBan [2014] 1) of Guangdong Province, China.

\section{References}

[1] C. Ballard, S. Gauthier, A. Corbett, C. Brayne, D. Aarsland, and E. Jones, "Alzheimer's disease," The Lancet, vol. 377, no. 9770, pp. 1019-1031, 2011.

[2] M. W. Weiner, "Dementia in 2012: further insights into Alzheimer disease pathogenesis," Nature Reviews Neurology, vol. 9, no. 2, pp. 65-66, 2013.

[3] P. Hollingworth, D. Harold, L. Jones, M. J. Owen, and J. Williams, "Alzheimer's disease genetics: current knowledge and future challenges," International Journal of Geriatric Psychiatry, vol. 26, no. 8, pp. 793-802, 2011.

[4] B. V. Zlokovic, "Neurovascular pathways to neurodegeneration in Alzheimer's disease and other disorders," Nature Reviews Neuroscience, vol. 12, no. 12, pp. 723-738, 2011.

[5] K. Bettens, K. Sleegers, and C. Van Broeckhoven, "Genetic insights in Alzheimer's disease," The Lancet Neurology, vol. 12, no. 1, pp. 92-104, 2013.

[6] K. Y. Chan, J. J. Wu, L. Liu et al., "Epidemiology of alzheimer's disease and other forms of dementia in China, 1990-2010: a systematic review and analysis," The Lancet, vol. 381, no. 9882, pp. 2016-2023, 2013.

[7] G. W. Beecham, K. Hamilton, A. C. Naj et al., "Genomewide association meta-analysis of neuropathologic features of Alzheimer's disease and related dementias," PLOS Genetics, vol. 10, no. 9, Article ID e1004606, 2014.

[8] J.-C. Lambert, C. A. Ibrahim-Verbaas, D. Harold et al., "Metaanalysis of 74,046 individuals identifies 11 new susceptibility loci for Alzheimer's disease," Nature Genetics, vol. 45, no. 12, pp. 1452-1458, 2013.

[9] S. Seshadri, A. L. Fitzpatrick, M. A. Ikram et al., "Genome-wide analysis of genetic loci associated with Alzheimer disease," Journal of the American Medical Association, vol. 303, no. 18, pp. 1832-1840, 2010.

[10] J. Mengel-From, K. Christensen, M. Thinggaard, M. Mcgue, and L. Christiansen, "Genetic variants in the choline acetyltransferase (ChAT) gene are modestly associated with normal cognitive function in the elderly," Genes, Brain and Behavior, vol. 10, no. 8, pp. 876-882, 2011.

[11] P. Davies and A. J. Maloney, "Selective loss of central cholinergic neurons in Alzheimer's disease," The Lancet, vol. 2, no. 8000, p. 1403, 1976.

[12] K.-W. Kim, Y.-J. Suh, W.-Y. Park et al., "Choline acetyltransferase $\mathrm{G}+4$ A polymorphism confers a risk for Alzheimer's disease in concert with Apolipoprotein E $\varepsilon 4$," Neuroscience Letters, vol. 366, no. 2, pp. 182-186, 2004.

[13] V. Mubumbila, A. Sutter, U. Ptok, R. Heun, and C. QuirinStricker, "Identification of a single nucleotide polymorphism in 
the choline acetyltransferase gene associated with Alzheimer's disease," Neuroscience Letters, vol. 333, no. 1, pp. 9-12, 2002.

[14] S. Ahn Jo, K. Ahn, J.-H. Kim et al., "ApoE- $\varepsilon$ 4-dependent association of the choline acetyltransferase gene polymorphisms (2384G $>$ A and $1882 \mathrm{G}>\mathrm{A}$ ) with Alzheimer's disease," Clinica Chimica Acta, vol. 368, no. 1-2, pp. 179-182, 2006.

[15] A. Ozturk, S. T. DeKosky, and M. I. Kamboh, "Genetic variation in the choline acetyltransferase (CHAT) gene may be associated with the risk of Alzheimer's disease," Neurobiology of Aging, vol. 27, no. 10, pp. 1440-1444, 2006.

[16] M. Tang, D. Rao, C. Ma et al., "Evaluation of choline acetyltransferase gene polymorphism (2384 G/A) in Alzheimer's disease and mild cognitive impairment," Dementia and Geriatric Cognitive Disorders, vol. 26, no. 1, pp. 9-14, 2008.

[17] E. Grünblatt, S. Zehetmayer, J. Bartl et al., "Genetic risk factors and markers for Alzheimer's disease and/or depression in the VITA study," Journal of Psychiatric Research, vol. 43, no. 3, pp. 298-308, 2009.

[18] E. Grünblatt, A. Reif, S. Jungwirth et al., "Genetic variation in the choline O-acetyltransferase gene in depression and Alzheimer's disease: the VITA and Milano studies," Journal of Psychiatric Research, vol. 45, no. 9, pp. 1250-1256, 2011.

[19] J. J. Lee, S. A. Jo, J. H. Park et al., "Choline acetyltransferase $2384 \mathrm{G}>$ a polymorphism and the risk of Alzheimer disease," Alzheimer Disease and Associated Disorders, vol. 26, no. 1, pp. 81-87, 2012.

[20] D. Harold, T. Peirce, V. Moskvina et al., "Sequence variation in the CHAT locus shows no association with late-onset Alzheimer's disease," Human Genetics, vol. 113, no. 3, pp. 258267, 2003.

[21] L. J. Cook, L. W. Ho, L. Wang et al., "Candidate gene association studies of genes involved in neuronal cholinergic transmission in Alzheimer's disease suggests choline acetyltransferase as a candidate deserving further study," American Journal of Medical Genetics Part B: Neuropsychiatric Genetics, vol. 132, no. 1, pp. 58, 2005.

[22] M. Piccardi, D. Congiu, A. Squassina et al., "Alzheimer's disease: Case-control association study of polymorphisms in ACHE, CHAT, and BCHE genes in a Sardinian sample," American Journal of Medical Genetics, Part B: Neuropsychiatric Genetics, vol. 144, no. 7, pp. 895-899, 2007.

[23] R. Scacchi, G. Gambina, G. Moretto, and R. M. Corbo, "Variability of AChE, BChE, and ChAT genes in the late-onset form of Alzheimer's disease and relationships with response to treatment with donepezil and rivastigmine," American Journal of Medical Genetics, Part B: Neuropsychiatric Genetics, vol. 150, no. 4, pp. 502-507, 2009.

[24] G. Wells, B. Shea, D. O'Connell et al., "The Newcastle-Ottawa Scale (NOS) for assessing the quality of nonrandomised studies in meta-analyses," 2016, http://www.ohri.ca/programs/clinical_ epidemiology/oxford.asp.

[25] W.-C. G. Yeung, W. D. Rawlinson, and M. E. Craig, "Enterovirus infection and type 1 diabetes mellitus: systematic review and meta-analysis of observational molecular studies," British Medical Journal, vol. 342, article d35, 2011.

[26] R. DerSimonian and N. Laird, "Meta-analysis in clinical trials," Controlled Clinical Trials, vol. 7, no. 3, pp. 177-188, 1986.

[27] N. Mantel and W. Haenszel, "Statistical aspects of the analysis of data from retrospective studies of disease," Journal of the National Cancer Institute, vol. 22, no. 4, pp. 719-748, 1959.

[28] J. P. Ioannidis, P. Boffetta, J. Little et al., "Assessment of cumulative evidence on genetic associations: interim guidelines,"
International Journal of Epidemiology, vol. 37, no. 1, pp. 120-132, 2008.

[29] Y.-Z. Song, H.-Y. You, Z.-H. Zhu et al., “The C825T polymorphism of the G-protein $\beta 3$ gene as a risk factor for functional dyspepsia: a meta-analysis," Gastroenterology Research and Practice, vol. 2016, Article ID 5037254, 11 pages, 2016.

[30] X. Xu, Y. Wang, L. Wang et al., "Meta-analyses of 8 polymorphisms associated with the risk of the Alzheimer's disease," PLoS ONE, vol. 8, no. 9, Article ID e73129, 2013.

[31] D. A. Evans, H. H. Funkenstein, M. S. Albert et al., "Prevalence of Alzheimer's disease in a community population of older persons. Higher than previously reported," Journal of the American Medical Association, vol. 262, no. 18, pp. 2551-2556, 1989.

[32] L. Letenneur, L. J. Launer, K. Andersen et al., "Education and the risk for Alzheimer's disease: sex makes a difference EURODEM pooled analyses," American Journal of Epidemiology, vol. 151, no. 11, pp. 1064-1071, 2000.

[33] F. Panza, A. D’Introno, A. M. Colacicco et al., "Vascular risk and genetics of sporadic late-onset Alzheimer's disease," Journal of Neural Transmission, vol. 111, no. 1, pp. 69-89, 2004.

[34] M. Zhang, R. Katzman, D. Salmon et al., "The prevalence of dementia and Alzheimer's disease in Shanghai, China: impact of age, gender, and education," Annals of Neurology, vol. 27, no. 4, pp. 428-437, 1990.

[35] C. A. Meier and T. D. Ong, "To feed or not to feed? A case report and ethical analysis of withholding food and drink in a patient with advanced dementia," Journal of Pain and Symptom Management, vol. 50, no. 6, pp. 887-890, 2015.

[36] G. Zhong, Y. Wang, Y. Zhang, J. J. Guo, and Y. Zhao, "Smoking is associated with an increased risk of dementia: a meta-analysis of prospective cohort studies with investigation of potential effect modifiers," PLoS ONE, vol. 10, no. 3, Article ID e0118333, 2015.

[37] Y. Huang and L. Mucke, "Alzheimer mechanisms and therapeutic strategies," Cell, vol. 148, no. 6, pp. 1204-1222, 2012.

[38] D. Krstic and I. Knuesel, "Deciphering the mechanism underlying late-onset Alzheimer disease," Nature Reviews Neurology, vol. 9, no. 1, pp. 25-34, 2013. 

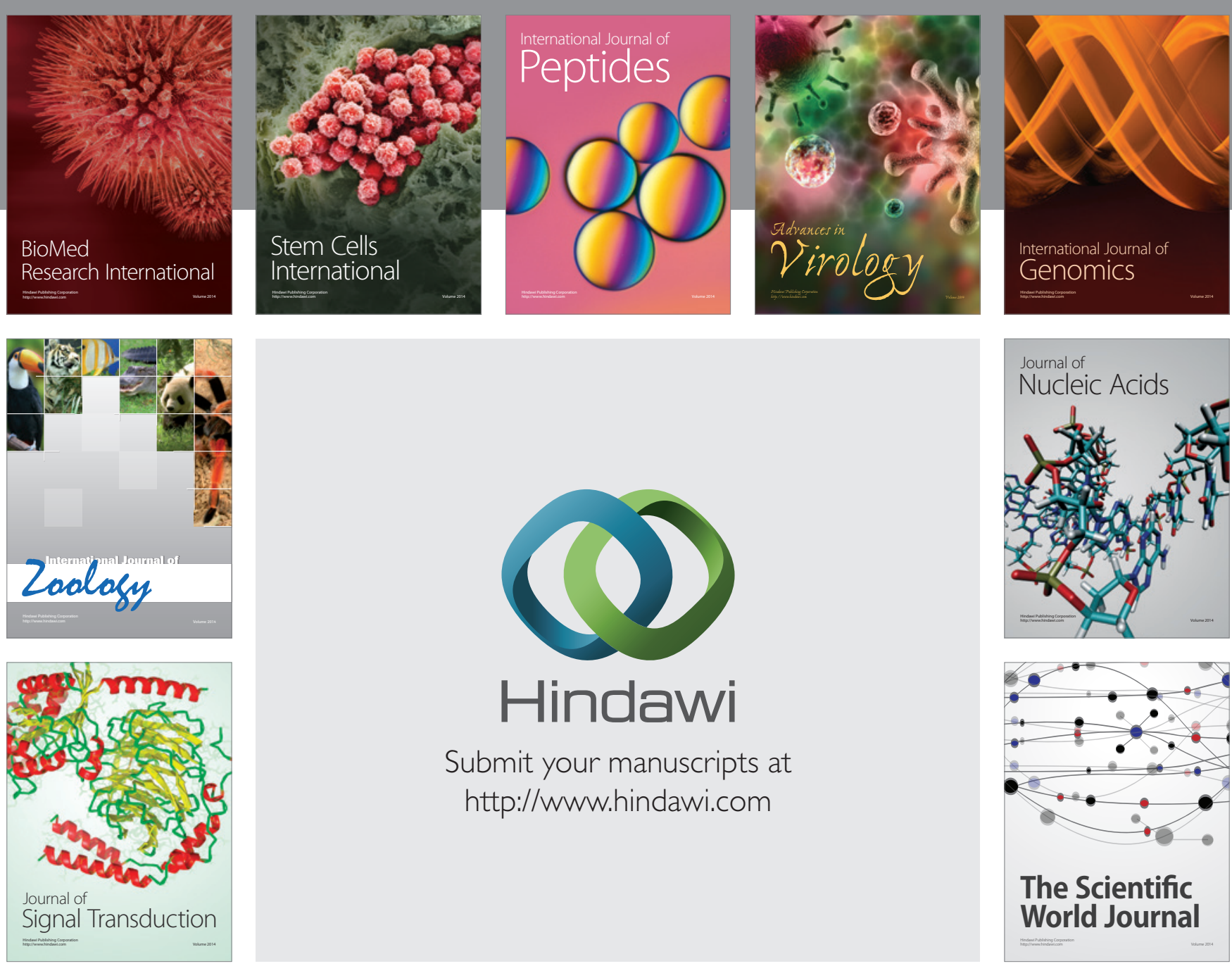

Submit your manuscripts at

http://www.hindawi.com
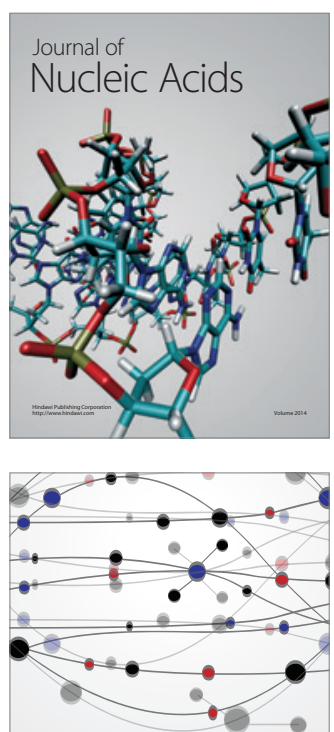

The Scientific World Journal
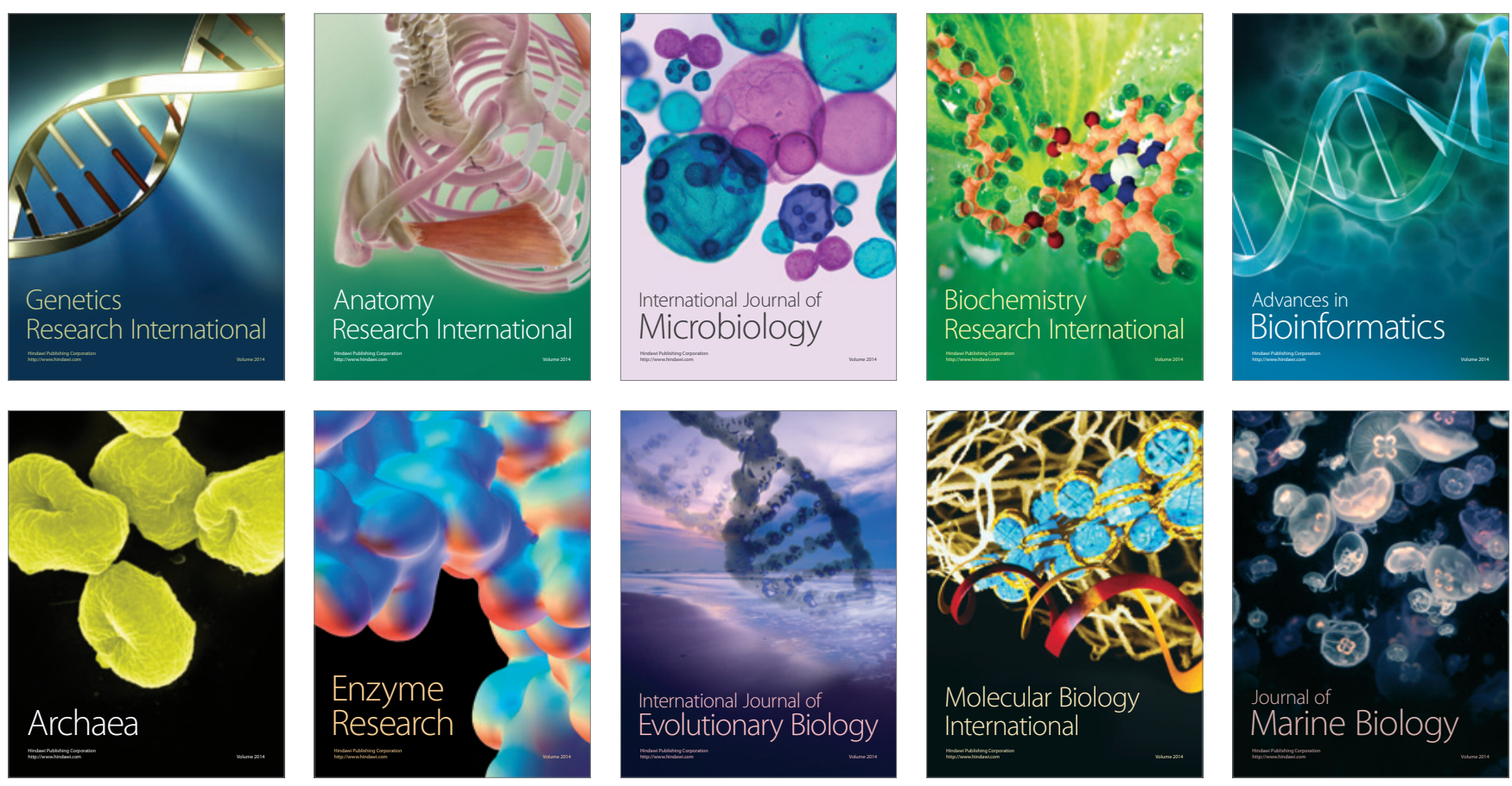